month mortality (aOR, 1.10; 95\% CI, 0.75-1.59; P = .635). The relationship between functional independence and symptom onset to door time comparing the two groups is shown in figure 1.

Discussion In patients selected for MT using non-contrast CT alone versus CT combined with CTA/MRA, we found improved procedural outcomes (shorten door-to-reperfusion time) and similar clinical outcomes and safety profiles. Our real-world findings indicate MT can be safely and effectively performed in hospitals lacking $24 / 7$ access to immediate CTA or MRA. The beneficial effect of using non-contrast CT-only may be present in patients with ultra-early symptoms to ED arrival (within the first hour) and should be further evaluated. Disclosures G. Ma: None. M. Mokin: None. B. Jia: None. A. P. Jadhav: None. X. Tong: None. D. Mo: None. N. Ma: None. F. Gao: None. X. Huo: None. G. Luo: None. L. Song: None. X. Sun: None. Y. Deng: None. A. Wang: None. X. Zhang: None. L. Gui: None. C. Song: None. Y. Peng: None. J. Wu: None. S. Zhao: None. J. Zhao: None. Z. Zhou: None. Z. Miao: None. Z. Ren: None.

\section{P-002 PERCEIVED LIMITS OF ENDOVASCULAR TREATMENT FOR SECONDARY MEDIUM VESSEL OCCLUSION STROKE}

${ }^{1} \mathrm{P}$ Cimflova, ${ }^{1} \mathrm{R}$ McDonough*, ${ }^{2} \mathrm{M}$ Kappelhof, ${ }^{3}$ J Ospel, ${ }^{4} \mathrm{~N}$ Singh, ${ }^{5} \mathrm{~N}$ Kashani, ${ }^{4} \mathrm{~A}$ Demchuk, ${ }^{4} \mathrm{~B}$ Menon, ${ }^{6} \mathrm{M}$ Chen, ${ }^{7} \mathrm{~N}$ Sakai, ${ }^{8} \mathrm{~J}$ Fiehler, ${ }^{1} \mathrm{M}$ Goyal. ${ }^{1}$ Department of Radiology, University of Calgary, Calgary, AB, Canada; 'Department of Radiology and Nuclear Medicine, University of Amsterdam, Amsterdam, Netherlands; ${ }^{3}$ Division of Neuroradiology, Clinic of Radiology and Nuclear Medicine, University Hospital Basel, Basel, Switzerland; ${ }^{4}$ Department of Clinical Neurosciences, University of Calgary, Calgary, $A B$, Canada; ${ }^{5}$ Department of Diagnostic Imaging, University of Calgary, Calgary, $A B$, Canada; ${ }^{6}$ Department of Neurological Sciences, Rush University Medical Center, Chicago, IL; 'Department of Neurosurgery, Kobe City Medical Centre General Hospital, Kobe, Japan; ${ }^{8}$ Department of Diagnostic and Interventional Neuroradiology, University Medical Center HamburgEppendorf, Hamburg, Germany

\subsection{6/neurintsurg-2021-SNIS.38}

Background Medium-vessel occlusions (MeVOs) account for $25 \%-40 \%$ of all acute ischemic stroke (AIS) cases. With periprocedural embolization occurring in up to $9 \%$ of all EVT cases, secondary MeVOs are of particular interest to neurointerventionalists. There is currently no reliable evidence regarding whether EVT is safe and effective for MeVO strokes. We sought to gain insight into the current management approaches regarding EVT in AIS caused by secondary MeVOs.

Methods We conducted an international case-based survey (MeVO-Finding Rationales and Objectifying New Targets for IntervEntional Revascularization in Stroke; MeVO-FRONTIERS) among stroke physicians to gain insight into the current management approaches regarding EVT in AIS caused by secondary MeVOs. Survey participants were presented three cases involving secondary $\mathrm{MeVO}$, each consisting of three case-vignettes with differences in the neurological status of the described patient (improvement, no change, unable to assess due to general anaesthesia). Univariate and multivariate logistic regression analyses clustered by respondent identity were performed to assess factors influencing the decision to treat.

Results 366 physicians (56 women, 308 men, 2 of undisclosed gender) of different specialities from 44 countries completed the survey for a total of 3294 responses. The majority of physicians $(54.1 \%, 1782 / 3294)$ were in favor of EVT. Participants were more likely to treat patients if the occlusion site was in the anterior $\mathrm{M} 2 / 3$ (74.3\%; risk ratio [RR] 2.62, 95\% CI:2.27-3.03) and A3 (59.7\%; RR 2.11, 95\%CI:1.83-2.42) segments, compared to those in the M3/4 segment $(28.3 \%$; reference). An improvement in neurological status led to a significant decrease in the likelihood to pursue EVT compared to patients whose neurological deficit remained unchanged $(49.9 \%$ versus $57 \%$ responses in favor of EVT, respectively; RR 0.88, 95\%CI:0.83-0.92). Interventionalists and more experienced physicians were more likely to treat secondary MeVOs.

Conclusion Our findings suggest that physician's willingness to treat secondary MeVOs endovascularly is limited and varies per occlusion location and change in neurological status. More evidence on the safety and efficacy of EVT for secondary MeVO stroke is needed.

Disclosures P. Cimflova: None. R. McDonough: None. M. Kappelhof: None. J. Ospel: None. N. Singh: None. N. Kashani: None. A. Demchuk: None. B. Menon: None. M. Chen: 2; C; Medtronic, Genentech, Stryker, Microvention, Cerenovus, Penumbra. N. Sakai: None. J. Fiehler: None. M. Goyal: 2; C; Mentice, Medtronic, Microvention, Stryker.

\section{P-003 ASSOCIATION BETWEEN BASELINE SEROLOGY AND SYMPTOMATIC INTRACRANIAL HEMORRHAGE AFTER ENDOVASCULAR THROMBECTOMY FOR ACUTE ISCHEMIC STROKE}

J Diestro*, C Parra-Farinas, M Balas, Z Zador, N Almusalam, A Dmytriw, P Rajendram, R Phillips, A Alqabbani, E Qazi, Y Li, W Montanera, D Sarma, T Marotta, A Bharatha, J Spears. St. Michael's Hospital, University of Toronto, Toronto, ON, Canada

\subsection{6/neurintsurg-2021-SNIS.39}

Background Intracranial hemorrhage after endovascular thrombectomy is associated with poorer prognosis compared to those who do not develop the complication. Our study aims to determine predictors of post-EVT hemorrhage - more specifically, inflammatory biomarkers present in baseline serology.

Methods We performed a retrospective review of consecutive patients treated with EVT for acute large vessel ischemic stroke. The primary outcome of the study is the presence of $\mathrm{ICH}$ on the post-EVT scan. We used four definitions: the SITS-MOST criteria, the NINDS criteria, asymptomatic hemorrhage and overall hemorrhage. We identified non-redundant predictors of outcome using backward elimination based on Akaike Information Criteria. We then assessed prediction accuracy using area under the receiver operating curve. Then we implemented variable importance ranking from logistic regression models using the drop in Naegelkerke $\mathrm{R}^{2}$ with the exclusion of each predictor.

Results Our study demonstrates a 6.3\% SITS (16/252) and $10.0 \%$ NINDS (25/252) sICH rate, as well as a $19.4 \%$ asymptomatic (49/252) and 29.4\% (74/252) overall hemorrhage rate. Serologic markers that demonstrated association with postEVT hemorrhage were: low lymphocyte count (SITS), high neutrophil count (NINDS, overall hemorrhage), low platelet to lymphocyte ratio (NINDS) and low total WBC (NINDS, asymptomatic hemorrhage).

Conclusion Our findings, particularly the association of diabetes mellitus and high neutrophil count with post-EVT hemorrhage, support experimental data on the role of thromboinflammation in hemorrhagic transformation of large vessel occlusions. 